\title{
EL SISTEMA EDUCACIONAL DE LA ARMADA DE CHILE
}

Dirección de Educación de la Armada de Chile 


\section{RESUMEN}

Los fundamentos de la educación naval y de la Armada de Chile se encuentran en los valores propios de la patria y de la nacionalidad -entendiéndose como tales los bienes humanos y físicos que la conforman, y el patrimonio espiritual, cultural e histórico-, y en las disposiciones establecidas en la Constitución Política de la República, como institución que forma parte del Estado.

La educación naval en Chile es un proceso intencionado que procura la formación integral del personal naval mediante las actividades de formación, instrucción y capacitación, las cuales son complementadas con las acciones de investigación y extensión. El sistema educacional es conducido por la Dirección de Educación de la Armada de Chile y llevado a cabo por las Academias, las Escuelas de Especialidades, las Escueles Matrices y los Centros de Instrucción.

\section{ABSTRACT}

The basis of naval education and of the Chilean Navy is to be found in the mother country's and nationality's principles: the human and physical aspects and the spiritual, cultural and historical inheritance that conform it, and in the rules established by the Republic's Political Constitution, since it is an institution that forms part of the State.

Chile's naval education is a process that seeks the staff's forming by means of training, instruction, all of which are complemented with investigation and other activities. The educational system is conducted by Chilean Naval's Educational Board (Dirección de Educación de la Armada de Chile) and is carried out by the Academies, the Specialities' Schools, the Master Schools and the Instruction Centres. 


\section{EL SISTEMA EDUCACIONAL DE LA ARMADA DE CHILE}

\section{FILOSOFÍA INSTITUCIONAL}

La Armada de Chile es heredera de una larga tradición que se integra dentro del desarrollo del país y, como institución de la defensa nacional, es esencial para ella.

Mediante la "Estrategia de los Tres Vectores", la Armada de Chile cumple las tareas que le impone su misión en el ámbito institucional, nacional e internacional, otorgando capacidades estratégicas reales al país y contribuyendo con la estrategia de seguridad nacional. El primer vector lo constituye la defensa directa de nuestro territorio, de sus ciudadanos, de sus bienes y de sus derechos; el segundo, la defensa y control de nuestro amplio espacio marítimo; el tercero se manifiesta en la participación de la Armada de Chile en la promoción y protección del interés nacional prioritario y fundamental: la conservación de la paz, la seguridad y la estabilidad del orden internacional en el mar, consustancial para nuestro desarrollo y progreso.

La Armada tiene una larga historia de servicio en tiempo de paz, materializada en la contribución entregada a los grandes desafíos nacionales de desarrollo, educación y colonización del territorio, entre otros, hasta un amplio espectro de acciones realizadas en apoyo de nuestra política exterior.

Fundamentos del sistema educacional naval

Es un hecho que la eficiencia y la eficacia de una organización descansan, principalmente, en la capacidad de sus hombres más que 
en sus medios. Por lo tanto, una de las preocupaciones principales de la Armada de Chile son las personas formadas, instruidas y entrenadas para que operen eficientemente los medios navales dispuestos bajo su responsabilidad. Esto requiere contar con un personal altamente motivado y preparado profesionalmente, capaz de desarrollar los diversos roles, necesarios para la planificación y ejecución con éxito de operaciones navales.

Esta preparación requiere liderazgo, especialización y constante perfeccionamiento, dado que los sistemas de armas navales son de avanzada tecnología y de evolución permanente. La Armada de Chile se encuentra a la vanguardia del desarrollo científico y tecnológico de los sistemas navales. Sus proyectos de diseño y construcción de sistemas y unidades navales son fundamentales para lograr una autonomía operativa; por eso entrega a su personal, en su etapa de especialización, las herramientas que le permiten mantenerse actualizado en su campo de acción

Las características propias de los sistemas navales hacen necesario que la preparación del personal sea realizada por la propia armada como un sistema cerrado, en cuanto a que genera y forma sus propios recursos humanos. Pero, al mismo tiempo, es abierta a los sistemas educacionales nacionales e internacionales.

La educación, en términos generales, es un proceso que debe ser asumido en dos dimensiones: como un proceso de asimilación moral y cultural y, al mismo tiempo, como un proceso de superación individual. Lo primero por cuanto, por su intermedio, las generaciones adultas, tanto en el sistema formal como informal, actúan sobre los educandos con la intención de que la persona en crecimiento llegue a identificarse y a participar de los bienes culturales, así como a integrarse en las formas sociales, costumbres y tradiciones predominantes en una comunidad. Debe entenderse como un proceso de culturización y adaptación, que permite preservar en cierto modo el "ser social". En virtud de esta intervención el sujeto adquiere el lenguaje, los criterios de valoración, las ideas científicas, las normas de comportamiento y los usos y formas sociales prevalecientes en su comunidad. Así, el educando se va integrando paulatinamente a su entorno de desarrollo y a quienes, directa o indirectamente, lo educan. 
La educación es, al mismo tiempo, un proceso de superación individual, puesto que intenta que el sujeto vaya desarrollando y haciendo efectivas sus propias potencialidades, que vaya descubriendo sus propias limitaciones y los tipos de actividades, relaciones y manifestaciones acordes con sus características personales. La educación, entendida en su esencia, debe responder a este doble intento, de estimular al educando para que vaya perfeccionando su capacidad de dirigir su propia vida y, al mismo tiempo, participar de su entorno.

Este concepto es el que asume la Armada de Chile ya que, considerando al hombre dentro de una dimensión singular que tiende a la perfectibilidad, lo hace partícipe de los bienes culturales, las costumbres y las tradiciones navales, de forma tal que, conscientemente, asuma su rol y contribuya al cumplimiento de nuestra misión.

En síntesis, la educación naval es un proceso que pretende el desarrollo integral de la persona. A través de la vida institucional se asumen los valores, la cultura, la ciencia y la técnica. Es una actividad intencionada, ética y permanente que procura modificar positivamente la conducta humana, principalmente mediante la interacción de las acciones de formación, instrucción y capacitación.

Las características de la educación naval

- Se inspira en los valores propios de la patria y de la nacionalidad, entendiéndose como tales los bienes humanos y físicos que la conforman y el patrimonio espiritual, cultural e histórico, de todos los chilenos.

- Utiliza para sus fines el campo del conocimiento humanista y científico universal, y el correspondiente a las áreas de las ciencias navales -aplicadas, sociales y humanas-, que se orientan hacia la conducción militar y la operación, administración y mantenimiento de los medios, como también hacia la investigación y el desarrollo tecnológico.

- Es continuadora y complementaria de la formación ciudadana que entrega oficialmente la educación pública y privada para 
aquellas personas que optan, libremente, por servir como hombres de armas a los intereses superiores de Chile. Los estudios se organizan de acuerdo con una jerarquía de especialización y capacitación según los roles que debe desempeñar el personal, desde la formación fundamental hasta la educación superior de posgrado.

- Es, por lo tanto, un proceso de acción completo e integral que educa a su personal en los ámbitos valórico, cognoscitivo y psicomotor, es decir, en los valores nacionales e institucionales, los conocimientos específicos propios de la profesión del hombre de armas y las destrezas intelectuales y físicas que demanda la actividad naval.

- Es un proceso flexible y dinámico que, mediante la acción de investigación, asimila el acelerado avance de la ciencia y la técnica, que ha hecho de la guerra naval un fenómeno de complejidad creciente.

- Como parte integral del sistema nacional de educación superior aporta, mediante la extensión, el conocimiento adquirido tanto en el ámbito académico institucional como extrainstitucional.

Los desafíos de la educación naval

El proceso educacional naval es conducido en forma integral por la Dirección de Educación de la Armada y, dentro de su misión para el próximo decenio, se ha planteado los siguientes desafíos:

- Determinar las necesidades de educación de la armada del futuro en cuanto a proveer y entregar a la institución personas con la actitud, compromiso, conocimientos y destrezas necesarios para satisfacer sus necesidades en los distintos ámbitos de desempeño profesional, teniendo en vista los proyectos de desarrollo del poder naval.

- Determinar las capacidades que se requerirán para cumplir con el propósito de planificar, ejecutar y evaluar el sistema educacional, utilizando armónicamente los aportes de las ciencias de la educación en aquellas áreas de excelencia que a la institución le interesan. 
- Entendiendo que la capacidad científica, tecnológica, cultural y valórica desarrollada con medios propios no es patrimonio exclusivo de la Armada de Chile, la institución está siempre en condiciones de contribuir a extender este cuerpo de conocimientos a otros componentes de la sociedad chilena. Esta actividad es cada día más dinámica por las tendencias de globalización que marcan el mundo de hoy y se proyectan hacia el futuro.

Por último, en la convicción de que el personal es el capital más importante de una organización, la Armada de Chile no sólo se esmera en formar, instruir y entrenar a su personal para servir eficazmente a la Patria, sino que también le entrega una alta calificación educacional y profesional que le permita contribuir significativamente a su desarrollo como personas y al mejoramiento de su calidad de vida.

Ante estos desafíos, el sistema educacional asume la responsabilidad de dar forma y ritmo al cambio y así definir el rumbo en torno a las finalidades esenciales del hombre. La ventaja comparativa que posee la armada, que permite que el sistema educacional naval se perfeccione y se preserve en el tiempo, es su capacidad de aprender, de actualizarse y de mantenerse a la vanguardia de la profesión naval.

\section{EL SISTEMA EDUCATIVO SUPERIOR DE LA INSTITUCIÓN}

La Dirección de Educación de la Amada es el organismo que tiene la misión de formar personal altamente calificado, tanto en los aspectos técnico-profesionales como valóricos y de fortaleza moral, necesarios para hacer frente a la guerra en el mar y a las múltiples misiones que tiene asignada la institución en tiempos de paz.

El primer nivel educacional se desarrolla en la Escuela Naval "Arturo Prat" -para quienes siguen la carrera de Oficial de Marina- y en las Escuelas de Grumetes y de Artesanos Navales -para quienes opten por integrarse como personal de Gente de Mar-. Los niveles de especialización y de posgrado se efectúan en las Academias Politécnicas y de Guerra, según corresponda. 
La Escuela Naval "Arturo Prat", ubicada en Valparaíso, imparte un programa de enseñanza de cuatro años al cabo de los cuales los cadetes egresan como guardiamarinas en alguno de los cuatro siguientes escalafones: Oficiales Ejecutivos e Ingenieros Navales, Infantes de Marina, Abastecimiento y Litoral. Los dos primeros años de estudio son comunes y los restantes corresponden a los del respectivo escalafón. Además, este plantel imparte educación a los cursos de Oficiales de Mar, Oficiales de Reserva y Oficiales de los Servicios de la Institución (Justicia, Sanidad Naval, Sanidad Dental y Servicio Religioso). (Ver cuadro $\mathrm{N}^{\mathrm{o}} 1$ )

La Escuela de Grumetes “Alejandro Navarrete Cisterna”, ubicada en la Isla Quiriquina, tiene por misión otorgar la formación fundamental e iniciar la capacitación a nivel técnico de nivel medio y técnico de nivel superior a los Grumetes Navales y a los Grumetes Infantes de Marina

Por su parte, la Escuela de Artesanos Navales, situada en Talcahuano, gradúa al personal de Gente de Mar (masculino y femenino) de los servicios, en los niveles de técnico de nivel medio y técnico de nivel superior, que se desempeñará en labores de mantención, abastecimiento, escribientes, sanidad naval y sanidad dental.

La educación naval especializada se imparte principalmente en la Academia Politécnica Naval, que posee tres facultades de reciente creación: Facultad de Sistemas de Armas (FASAR), Facultad de Sistemas de Ingeniería y Logísticas (FASILOG) y Facultad de Sistemas de Apoyo Operativo (FASAP). También se entrega este tipo de educación en la Escuela de Aviación Naval, en la Escuela de Submarinos y en los cursos que se imparten en el Servicio Hidrográfico y Oceanográfico de la Armada; además en el Comando de Buzos Tácticos y en el "Buque Escuela Esmeralda".

Todos los alumnos egresados de la Escuela de Grumetes "Alejandro Navarrete Cisterna" deben completar su formación en la Academia Politécnica Naval, donde obtienen el título técnico de nivel medio o superior en menciones como Artillería, Torpedos y Operador de Sonar, Navegación, Operación de Radares, Electromecánica, Mecánica Electrónica, Mecánica de Combustión Interna y 
Telecomunicaciones. Por su parte, los soldados Infantes de Marina, al egresar de la Academia Politécnica Naval, obtienen título técnico de nivel medio o superior en menciones como Armas Anfibias, Mecánico de Material de Guerra y Mecánico Electrónico, entre otras. (Ver cuadros $\mathrm{N}^{\circ} 2 \mathrm{y} \mathrm{N}^{\circ} 3$ )

Una vez titulados, los marineros y los soldados de Infantería de Marina deben asumir funciones a bordo o en tierra por alrededor de doce años, aplicando sus conocimientos y adquiriendo destrezas a medida que asumen nuevas y mayores responsabilidades. En esta etapa, el personal debe cumplir con el requisito de perfeccionamiento flexible, que considera como válidos todas las capacitaciones y los estudios realizados con posterioridad a los cursos de especialidad, tanto en la institución como en organismos extrainstitucionales.

En la medida en que adquieren mayor experiencia, van ascendiendo en la escala jerárquica y accediendo a cursos de capacitación superior que permiten a los beneficiados obtener la autoridad técnica necesaria para asumir responsabilidades de mando y conducción de las generaciones más jóvenes.

En el caso de los oficiales de línea, la educación especializada comienza cuando los cadetes ingresan a uno de los cuatro escalafones, continúa durante el crucero de instrucción a bordo del "Buque Escuela Esmeralda" y culmina en la Academia Politécnica Naval. Existen especialidades que demandan cinco años de estudios y otras seis, lo que se ve reflejado en el título profesional y grado académico que reciben los oficiales.

La educación naval superior para oficiales continúa tanto en la Academia Politécnica Naval como en la Academia de Guerra Naval o en institutos de educación superior nacionales y extranjeros. (Ver cuadro $\mathrm{N}^{\circ} 4$ )

La Academia de Guerra Naval es el plantel educacional encargado de desarrollar las actividades educacionales del más alto nivel en el campo de la docencia e investigación de las ciencias y artes militares, así como en las ciencias sociales y económicas relacionadas, y en aquellas destinadas a perfeccionar las capacidades de los oficiales alumnos. Para ello se imparten cursos y programas orientados al 
perfeccionamiento de Oficiales de Línea y también de profesionales del ámbito civil. Entre los primeros figura el Curso de Estado Mayor, destinado a formar oficiales para desempeñarse en altos puestos de mando y de asesoría operativa y administrativa, tanto institucionales como conjuntos.

Además, se dicta el Curso de Informaciones para Oficiales de Servicios Generales y Oficiales de los Servicios Especializados que contiene los principios básicos de las distintas funciones de Estado Mayor y otros aspectos relacionados con las operaciones navales y la política de defensa nacional, con el fin de que los egresados puedan asesorar al mando en materias relacionadas con su especialidad y contribuir al mejor empleo de los medios institucionales. La Academia de Guerra Naval imparte también un Programa de Magíster en Ciencia Política Integrada, en convenio con la Universidad Marítima de Chile, dirigido a oficiales de las Fuerzas Armadas y de Orden y a profesionales civiles de las más diversas áreas del quehacer regional y nacional. Dentro de sus actividades académicas, en forma permanente, realiza programas de diplomados sobre temas relevantes de interés institucional, incluyendo también a personeros del sector público y privado del país.

\section{SISTEMA DE FORMACIÓN Y PROGRAMAS DE ESTUDIOS}

La educación naval está plenamente integrada al sistema de educación nacional y el Estado le otorga reconocimiento legal a todas sus academias y escuelas a través de la Ley Orgánica Constitucional de Enseñanza (LOCE), quedando facultada para otorgar títulos técnicos de nivel superior, títulos profesionales y grados académicos.

Los cuadros siguientes describen el orden en que se otorgan títulos y grados. 


\section{CUADRO $\mathrm{N}^{0} 1$}

Títulos profesionales de oficiales ejecutivos, ingenieros navales, infantería de marina, abastecimiento y litoral

\begin{tabular}{|l|l|}
\hline Especialidad & Título profesional \\
\hline Guerra de Superficie & Ingeniero naval de armamentos \\
\hline Guerra Antisubmarina & Ingeniero naval de armamentos \\
\hline Guerra Electrónica & Ingeniero naval electrónico \\
\hline Guerra Electrónica de Telecomunicaciones & Ingeniero naval electrónico \\
\hline Guerra Aeronaval & Ingeniero naval de armamentos \\
\hline Guerra Submarina & Ingeniero naval de armamentos \\
\hline Hidrografía y Oceanografía & Ingeniero naval en hidrografía y oceanografía \\
\hline Meteorología & Ingeniero de ejecución en meteorología \\
\hline Administración Naval & Ingeniero de ejecución en administración naval \\
\hline Ingeniería Naval Mecánica & Ingeniero naval mecánico \\
\hline Ingeniería Naval Eléctrica & Ingeniero naval electricista \\
\hline Salvataje y Control de Averías & Ingeniero de ejecución en salvataje y control de averías \\
\hline $\begin{array}{l}\text { Guerra Electrónica complementada en: } \\
\text { Aviación Naval Submarinos }\end{array}$ & $\begin{array}{l}\text { Ingeniero naval electrónico mención en: } \\
\text { aeronáutica submarinos }\end{array}$ \\
\hline $\begin{array}{l}\text { Ingeniería Naval Mecánica complementada en: } \\
\text { Aviación Naval Submarinos }\end{array}$ & $\begin{array}{l}\text { Ingeniero naval mecánico mención en: } \\
\text { aeronáutica submarinos }\end{array}$ \\
\hline Ingeniería Naval Eléctrica complementada en: & Ingeniero naval electricista mención en: \\
\hline Aviación Naval Submarinos & aeronáutica submarinos \\
\hline Infantería de Marina & Ingeniero en sistemas anfibios \\
\hline Abastecimiento & Ingeniero en abastecimiento naval \\
\hline Litoral & Ingeniero en administración marítima \\
\hline
\end{tabular}

\section{CUADRO $\mathrm{N}^{0} 2$}

Títulos técnicos de nivel medio.

Personal de gente de mar de línea

\begin{tabular}{|l|l|}
\hline Especialidad & $\begin{array}{l}\text { Título técnico de nivel medio en: } \\
\text { Área de Armamentos }\end{array}$ \\
\hline Artillería & Armas, mención artillería \\
\hline Torpedista Operador de Sonar & Armas, mención torpedos y operador de sonar \\
\hline Maniobras & Maniobras náuticas \\
\hline Buceo Táctico & Buceo táctico \\
\hline & Área de Operaciones \\
\hline Comunicaciones & Operación de sistemas de comunicaciones \\
\hline Radar CIC & Operación de radares \\
\hline Navegación & Navegación \\
\hline & Área de Infantería de Marina \\
\hline Infantería & Infantería, mención infantería de marina \\
\hline Artillería IM & Artillería, mención Infantería de marina \\
\hline Ingeniero de Combate & Ingeniero de combate, mención infantería de marina \\
\hline
\end{tabular}




\begin{tabular}{|l|l|}
\hline Especialidad & Título técnico de nivel medio en: \\
\hline Abastecimiento & Área de Abastecimiento \\
\hline Escribiente & Abastecimiento \\
\hline Cocinero & Secretaría administrativa, mención computación \\
\hline Mayordomo & Artes culinarias \\
\hline & Administración, servicio y repostería \\
\hline Litoral & Área de Litoral \\
\hline & Administración marítima \\
\hline Meteorología & Área de Meteorología \\
\hline Personal de Gente de Mar de los Servicios. & Meteorología \\
\hline & \\
\hline Chofer Mantención Automotriz & Área de Mantenimiento y Operación \\
\hline & Mantención automotriz \\
\hline Abastecimiento & Área de Abastecimiento \\
\hline Escribiente & Abastecimiento \\
\hline Guardalmacén & Secretaría administrativa, mención computación \\
\hline & Guardalmacén \\
\hline Sanidad Naval & Área de Sanidad \\
\hline Sanidad Dental & Auxiliar de enfermería \\
\hline
\end{tabular}

\section{CUADRO N $\mathrm{N}^{\circ} 3$}

Títulos técnicos de nivel superior

Personal de gente de mar de línea

\begin{tabular}{|l|l|}
\hline Especialidad & $\begin{array}{l}\text { Título técnico de nivel superior } \\
\text { Área de Armamentos }\end{array}$ \\
\hline Mecánico Artillero & $\begin{array}{l}\text { Mecánica, mención en mantenimiento de máquinas y } \\
\text { equipos hidroneumáticos }\end{array}$ \\
\hline Mecánico Electrónico Control de Fuego & $\begin{array}{l}\text { Mecánica electrónica, mención mantenimiento equipos } \\
\text { electrónicos }\end{array}$ \\
Mecánico Electrónico Control de Fuego & $\begin{array}{l}\text { Mecánica electrónica, mención mantenimiento equipos } \\
\text { electrónicos }\end{array}$ \\
\hline $\begin{array}{l}\text { Mecánico Electrónico Radar Control de } \\
\text { Fuego y Designadores }\end{array}$ & $\begin{array}{l}\text { Mecánica electrónica, mención mantenimiento equipos y } \\
\text { electrónicos }\end{array}$ \\
\hline Mecánico Electrónico Sonar & $\begin{array}{l}\text { Mecánica electrónica, mención mantenimiento equipos } \\
\text { electrónicos }\end{array}$ \\
\hline Mecánico Electrónico en Guerra Electrónica & $\begin{array}{l}\text { Mecánica electrónica, mención mantenimiento equipos } \\
\text { electrónicos }\end{array}$ \\
\hline Mecánico Electrónico en Telecomunicaciones & $\begin{array}{l}\text { Mecánica electrónica, mención mantenimiento equipos } \\
\text { electrónicos }\end{array}$ \\
Mecánico Electrónico en Radares & $\begin{array}{l}\text { Mecánica electrónica, mención mantenimiento equipos } \\
\text { electrónicos }\end{array}$ \\
\hline Mecánico Electrónico en Navegación & $\begin{array}{l}\text { Mecánica electrónica, mención mantenimiento equipos } \\
\text { electrónicos }\end{array}$ \\
\hline
\end{tabular}




\begin{tabular}{|c|c|}
\hline \multirow[t]{2}{*}{ Especialidad } & Título técnico de nivel superior \\
\hline & Área de Ingeniería \\
\hline Mecánico de Máquinas & Mecánica en maquinaria naval \\
\hline Mecánico de Combustión Interna & Mecánica en combustión interna \\
\hline Mecánico Control de Averías & Mecánica en control de averías \\
\hline Mecánico Electricista & Electromecánica, mención sistemas de poder \\
\hline \multirow[t]{2}{*}{ Buceo de Salvataje Control de Averías } & Buceo de salvataje, mención control de averías \\
\hline & Área de Aviación Naval \\
\hline \multirow[t]{2}{*}{ Mecánico Aviación } & Mecánica en aviación \\
\hline & Área de Infantería de Marina \\
\hline \multirow[t]{2}{*}{ Mecánico Material de Guerra } & $\begin{array}{l}\text { Mecánica en material de guerra, mención infantería de } \\
\text { marina }\end{array}$ \\
\hline & Área de Sanidad \\
\hline Enfermero & Enfermería \\
\hline Técnico de Sumersión & Enfermería de sumersión \\
\hline Técnico en Enfermería Aerospacial & Enfermería aerospacial \\
\hline \multirow[t]{2}{*}{ Enfermero Técnico de Apoyo Clínico en: } & Enfermería, mención en: \\
\hline & Pabellón \\
\hline Pabellón & Anestesia \\
\hline Anestesia & Banco de sangre \\
\hline Banco de Sangre & Laboratorio \\
\hline Laboratorio & Rayos "X" \\
\hline Rayos "X" & Salud Pública e Higiene Ambiental \\
\hline Medicina Preventiva & Odontología \\
\hline \multicolumn{2}{|l|}{ Odontología } \\
\hline \multicolumn{2}{|l|}{ Personal de Gente de Mar de los Servicios } \\
\hline \multirow[t]{2}{*}{ Especialidad } & Título Técnico de Nivel Superior en: \\
\hline & Área de Mantenimiento y Operación \\
\hline Artillería & Armas, mención artillería \\
\hline Artificiero & Explosivos \\
\hline Electrónico & Electrónica \\
\hline Máquinas & Maquinaria naval \\
\hline Electricista & Electricista \\
\hline Combustión Interna & Combustión interna \\
\hline Calderas & Calderas \\
\hline Metalurgia & Metalurgia \\
\hline Operador de Máquinas y Herramientas & Operación de máquinas y herramientas \\
\hline Estructuras Metálicas & Estructuras metálicas \\
\hline Cañerías & Cañerías de alta presión \\
\hline Soldador & Soldadura industrial \\
\hline Carpintería & Carpintería \\
\hline Dibujante & Dibujo técnico \\
\hline Artes Gráficas & Artes gráficas \\
\hline Hidrografía y Oceanografía & Hidrografía y oceanografía \\
\hline
\end{tabular}




\section{CUADRO N 4}

Grados académicos de los oficiales ejecutivos, ingenieros navales, infantería de marina, abastecimiento y litoral

\begin{tabular}{|l|l|}
\hline Grado Académico & Mención \\
\hline Licenciado en "Ciencias Navales y Marítimas", & Guerra de superficie \\
\hline & Guerra submarina \\
\hline & Guerra antisubmarina \\
& Guerra electrónica \\
\hline & Guerra aeronaval \\
\hline & Hidrografía y oceanografía \\
\hline & Ingeniería naval mecánica \\
\hline & Ingeniería naval eléctrica \\
\hline & Ingeniería naval electrónica \\
\hline & Ingeniería naval mecánica, eléctrica o electrónica, \\
& mención aeronáutica \\
\hline & Infantería de marina \\
\hline & Abastecimiento \\
\hline Magíster en "Ciencias Navales y Marítimas", & Litoral \\
\hline & Estrategia \\
\hline & Geopolítica \\
\hline & Oceanopolítica \\
\hline & Operaciones anfibias \\
\hline & Táctica \\
\hline & Logística \\
\hline
\end{tabular}

